\title{
White matter hypoplasia-corpus callosum agenesis-intellectual disability syndrome
}

INSERM

\section{Source}

INSERM. (1999). Orphanet: an online rare disease and orphan drug data base. White matter hypoplasia-corpus callosum agenesis-intellectual disability syndrome.

ORPHA:3207

A rare, genetic, multiple cong enital anomalies/dysmorphic syndrome characterized by severe white matter hypoplasia, corpus callosum agenesis or extreme hypoplasia, severe intellectual disability, failure to thrive and minor midline facial dysmorphism (including hypertelorism, broad nasal root, microg nathia). There have been no further descriptions in the literature since 1993. 